\title{
Control of Gas Dehydration Unit Using Multivariable Model Predictive Control (MMPC) to Obtain More Optimal Control Performance
}

\author{
Abdul Wahid ${ }^{1, *}$, Rickson Mauricio ${ }^{1}$ and Naufal Syafiq Maro ${ }^{1}$ \\ ${ }^{1}$ Chemical Engineering Department, Universitas Indonesia, Depok, Indonesia
}

\begin{abstract}
A multivariable model predictive control (MMPC) is proposed to improve a control performance in Gas dehydration process. The FOPDT models are used to build an MMPC derived from the selected controlled variables (CV) and manipulated variables (MV). A set point (SP) tracking is used to test the control performance, with proportional-integral controller ( $\mathrm{PI})$ as a comparison. As an indicator of the control performance is the integral of square error (ISE). The result is a TITO (two-inputs two-outputs) MMPC, with sweet gas flow rate and heat duty of heater as MVs, and feed pressure and heater temperature as CVs, respectively. In the SP tracking test, MMPC showed better control performance than the PI controller with $11.29 \%$ performance improvement (pressure control) and $16.39 \%$ (temperature control).
\end{abstract}

\section{Introduction}

In the exploration industry in Indonesia, besides being produced by petroleum, natural gas is also produced which will be sold to industries engaged in gas management. Natural gas is a fossil fuel that is odorless, shapeless, has a large flower power, high-pressure, low specific gravity, is a non-renewable resource and can produce large amounts of energy [1]. Natural gas generally collects below the soil layer, containing various compositions contained in the oil content or associated gas. All that exists in the petroleum content is related to natural gas, which is dissolved in crude oil and often forms gas caps or gas caps on oil content [2]. Natural gas can also gather in coal mines and in natural gas fields. The main component in natural gas is methane $\left(\mathrm{CH}_{4}\right)$, which is the shortest and lightest chain hydrocarbon molecule. Natural gas also contains heavier hydrocarbon molecules such as ethane $\left(\mathrm{C}_{2} \mathrm{H}_{6}\right)$, propane $\left(\mathrm{C}_{3} \mathrm{H}_{8}\right)$ and butane $\left(\mathrm{C}_{4} \mathrm{H}_{10}\right)$, as well as gases containing sulfur (sulfur). Table 1. shows the natural gas composition in general.

Table 1. Natural Gas Composition [2]

\begin{tabular}{lc}
\hline \multicolumn{1}{c}{ Component } & Percentage \\
\hline Methane $\left(\mathrm{CH}_{4}\right)$ & $80-90$ \\
\hline Ethane $\left(\mathrm{C}_{2} \mathrm{H}_{6}\right)$ & $5-15$ \\
\hline Propane $\left(\mathrm{C}_{3} \mathrm{H}_{8}\right)$ and Butane $\left(\mathrm{C}_{4} \mathrm{H}_{10}\right)$ & $<5$ \\
\hline
\end{tabular}

*Corresponding author: wahid@che.ui.ac.id
Natural gas originating from reservoir has not met specifications for gas sales even for LNG (Liquefied Natural Gas). Therefore, before becoming a gas sales or $\mathrm{LNG}$, it is necessary to do the processing processes on the natural gas. In general, before the gas dehydration process there are two processing process done first, namely the process of gas sweetening and sulfur recovery. The sweetening gas process is carried out to remove the acid content of the natural gas, in example hydrogen sulfide $\left(\mathrm{H}_{2} \mathrm{~S}\right)$ and carbon dioxide $\left(\mathrm{CO}_{2}\right)$. Meanwhile, the sulfur recovery process is done to reduce sulfur content in natural gas. After that, then the gas dehydration process is done on the gas dehydration unit to remove the water content in natural gas to a minimum. Thereafter, a mercury removal process and a fractionation process are conducted to separate the $\mathrm{C} 1$ component with the heavier fraction $(\mathrm{C} 2+)$.

In natural gas management, it is expected to become a dry gas. Gas dehydration process is one of the most common process. The presence of hydrocarbon and water vapor content will lead to the formation of hydrates in the form of crystals and corrosive. Hydrate formation can reduce pipeline flow capacity, even cause blockage, and potential damage to the filter, valve and process compressor. Hydrate is a combination of excess water vapor with liquid hydrocarbons, which can condense from gases in the course of transmission, to form emulsions which under pressure conditions are solid mass processes [3]. One way to prevent the occurrence of hydrate is to reduce or even eliminate the water content in natural gas until the water content is 
very small. Since the water content of natural gas when taken from the reservoir reaches 902.1 ppm (part per million) or well above the gas sales standard of 7 $\mathrm{lb} / \mathrm{MMSCF}$ or about $150 \mathrm{ppm}$, before being sold as a gas sales, natural gas must be processed first by drying through the gas dehydration unit. The specification of gas sales content to be sent using pipes in the process must meet some specifications that have been determined according to company standards, by looking at several factors namely pressure, temperature, flow rate, and other specifications such as maximum water content, hydrocarbon content, and other concentrations such as mercaptan, $\mathrm{CO}_{2}$, and the minimum heating value [4]. But the problem is that although natural gas has been dried, the water content contained in the product gas sales is still above $7 \mathrm{lb}$ /MMSCF. This becomes a problem because when gas is sent by pipeline from the gas processing industry to the consumer, it will form a gas hydrate that will cause the depreciation of the amount (volume) of gas received by the consumer.

Abdulrahman and Sebastine [5] have simulated a natural gas dehydration process of Khurmala field based on steady-state mode using Hysys so that it does not apply the control system. Therefore, in this study, the dehydration process that is located in Terminal $\mathrm{X}$ is simulated in dynamic mode and applies the control system in it. The most dominant control system used in the industry is the PI controller because it is easier to understand and operate it [6-7]. However, nonlinearities and inter-process-variable interactions can not be overcome by PI controllers. Therefore, MMPC is the most appropriate solution [8-11].

\section{Methodology}

The natural gas to be processed in the dehydration unit is located at Terminal $\mathrm{X}$ with the feed specifications shown in the Table 3 and the parameters used in performing the process simulation are shown in Table 3. Triethylene Glycol (TEG) is utilized as a liquid absorbent to absorb moisture content from the sweet gas stream.

Table 2. Feed Gas Specificaton

\begin{tabular}{lr}
\hline \multicolumn{1}{c}{ Component } & \%Mole \\
\hline Methane & 88.921 \\
\hline Ethane & 3.809 \\
\hline Propane & 3.144 \\
\hline i-Butane & 0.59 \\
\hline n-Butane & 0.863 \\
\hline i-Pentane & 0.25 \\
\hline n-Pentane & 0.17 \\
\hline n-Hexane & 0.349 \\
\hline Water & 1.9 \\
\hline Carbon dioxide & 0.004 \\
\hline Nitrogen & trace \\
\hline Hydrogen sulfide & trace \\
\hline
\end{tabular}

Table 3. Parameters required for conducting simulations

\begin{tabular}{lr}
\hline \multicolumn{1}{c}{ Parameter } & Value \\
\hline Feed gas flow rate (MMSCFD) & 70 \\
\hline Pressure operation of contactor $(\mathrm{psi})$ & 840.7 \\
\hline Reflux ratio of regenerator & 0.01 \\
\hline Temperature operation of reboiler $\left({ }^{\circ} \mathrm{C}\right)$ & 193.3 \\
\hline Lean glycol purity $(\%)$ & 97.4 \\
\hline Lean glycol circulation rate (USGPM) & 25 \\
\hline Pump adiabatic efficiency $(\%)$ & 68 \\
\hline
\end{tabular}

Steady state simulation is a flow simulation in the production process without considering the disturbance with time. This simulation is used to ensure that the process is correct and can run. All parameters that should be fluid and need to be controlled, are considered constant. Steady state simulations are deemed to be completed if the sales gas specification is appropriate. Changing the steady state simulation to a dynamic process is made to estimate product results that depend on the time function and to see the effect of disturbance with the operating conditions of the process.

Then the dynamic behavior of the process is modeled by the first order plus dead time model (FOPDT) due to the limitations of UniSim software which only accommodates the FOPDT model when MPC used as a controller. The FOPDT parameters (Kp, $\tau, \theta)$ are then fed into the MMPC. The system model identification procedure [12] is performed by varying the valve opening changes to obtain a system model that provides the best MMPC control results.

The control system is identified by installing the proper controller. The controller to be installed is a temperature control on the heater and the pressure control on the contactor. In the installation of the controller, the thing to consider is the control objective.

Based on the model obtained from PRC (Process Reaction Curve), tuning is performed to obtain optimal control parameters. In Multivariable MPC, tuning parameter is $\mathrm{T}$ (sample time), $\mathrm{P}$ (prediction horizon), and $\mathrm{M}$ (control horizon). The method used is the result of calculation with the development of Shridhar and Cooper [13]. From the values of $\mathrm{Kp}, \tau$, and $\theta$, obtained the calculated values of $\mathrm{T}, \mathrm{P}$, and $\mathrm{M}$ by using the equations.

However, if the method does not succeed in getting the desired product quality, then re-identification system to get the new empirical process model so as to get the new $\mathrm{Kp}, \tau$, and yang value which is then recalculated Multivariable MPC control parameters. But if you do not get the right control parameters, can use fine tuning method based on the parameters that have been obtained before.

\section{Results and discussion}

The objective of the parametric study is to determine the appropriate controls used in order to be able to control the entire operation process on the gas dehydration unit so as to obtain sales gas in accordance 
with the specification. The following section presents the results.

\subsection{Design of Gas Dehydration Unit}

In the simulation process of the dehydration unit of this gas, the feed which is at once the output or output of the previous unit is Acid Gas Removal has a flow rate of 70 MMSCFD, with a temperature of $20^{\circ} \mathrm{C}$, and a pressure of 840.7 psi. The feed enters at the bottom of the absorber column under high pressure and low temperature operating conditions, then contacts with a high purity (above 90\%) glycol (Triethylene Glycol) solvent which enters the top of the absorber column. The design specification of the column consists of 10 stages with the type of tray used is sieve tray.

The result of direct contact on the absorber column is dry gas which is then flowed through the heat exchanger to raise the temperature up to $35.84^{\circ} \mathrm{C}$ to match the specifications of the established gas sales. Then, the glycol solvent which has absorbed moisture in the feed stream (rich TEG) is regenerated in the regenerator column at a reboiler temperature of $193.3^{\circ} \mathrm{C}$, and with the operating conditions of the regenerator column in example high temperature and low pressure. The column specification design consists of 5 stages with internal column design using packing type.

Simulation results on steady state conditions can be seen in Table 4. It is seen that the water content in the gas sales has reached the standard limit set. In addition, it is also seen that the composition of methane in gas sales is higher than the gas flow of the feed (sweet gas).

Table 4. Sales Gas Specification in Steady State Condition

\begin{tabular}{ll}
\hline \multicolumn{1}{c}{ Specification } & \multicolumn{1}{c}{ Value } \\
\hline Water Content & $5.48 \mathrm{lb} / \mathrm{MMSCF}$ \\
\hline Heating Value & $1037 \mathrm{Btu} / \mathrm{SCF}$ \\
\hline Temperature & $43.54^{\circ} \mathrm{C}\left(120.38^{\circ} \mathrm{F}\right)$ \\
\hline Pressure & $832 \mathrm{psi}(817.3 \mathrm{psig})$ \\
\hline Flow Rate & $68.65 \mathrm{MMSCFD}$ \\
\hline Methane Composition & $90.63 \%($ mole $)$ \\
\hline
\end{tabular}

The design of the control on the gas dehydration unit in the gas processing as a whole uses TITO (twoinputs two-outputs) system, ie., sweet gas flow rate and heat duty of heater as MVs (see Table 5), and feed pressure and heater temperature as $\mathrm{CVs}$, respectively. The process control system of the gas dehydration unit is shown in Figure 1.

Table 5. TITO MMPC

\begin{tabular}{ccc}
\hline $\begin{array}{c}\text { Type of } \\
\text { Controller }\end{array}$ & $\begin{array}{c}\text { Controlled } \\
\text { Variable }\end{array}$ & $\begin{array}{c}\text { Manipulated } \\
\text { Variable }\end{array}$ \\
\hline Pressure & Feed gas & Sweet gas flow \\
Control & pressure & rate \\
\hline Temperature & Temperature & Heater duty \\
Control & out from heater & \\
\hline
\end{tabular}

\subsection{Identification of Systems with Empirical Modeling Processes}

The ability of the MPC controller to stabilize the process depends on the empirical model of a process used for tuning. The model used in this research is empirical model with First Order Plus Dead Time (FOPDT) approach. The FOPDT empirical model of a process is obtained by performing a model testing on the control simulation which is then obtained by a Process Reaction Curve (PRC). This PRC will provide the values needed to perform tuning on the Multivariable MPC controllers. Type of testing model used is open loop. Empirical values $(\mathrm{Kp}, \tau, \theta)$ are used as control action parameters on the MPC controller.

The system identification was performed to obtain a dynamic model of gas dehydration process. The system identification is done by changing the amount of manipulated variable (in this case is the feed rate / sweet gas and heat heater load) with step change in open loop process. Changes in the value of controlled variables due to changes in manipulated variables until a certain time interval can be described in PRC. The dynamic behavior of processes described in the PRC can be modeled with the FOPDT empirical model.

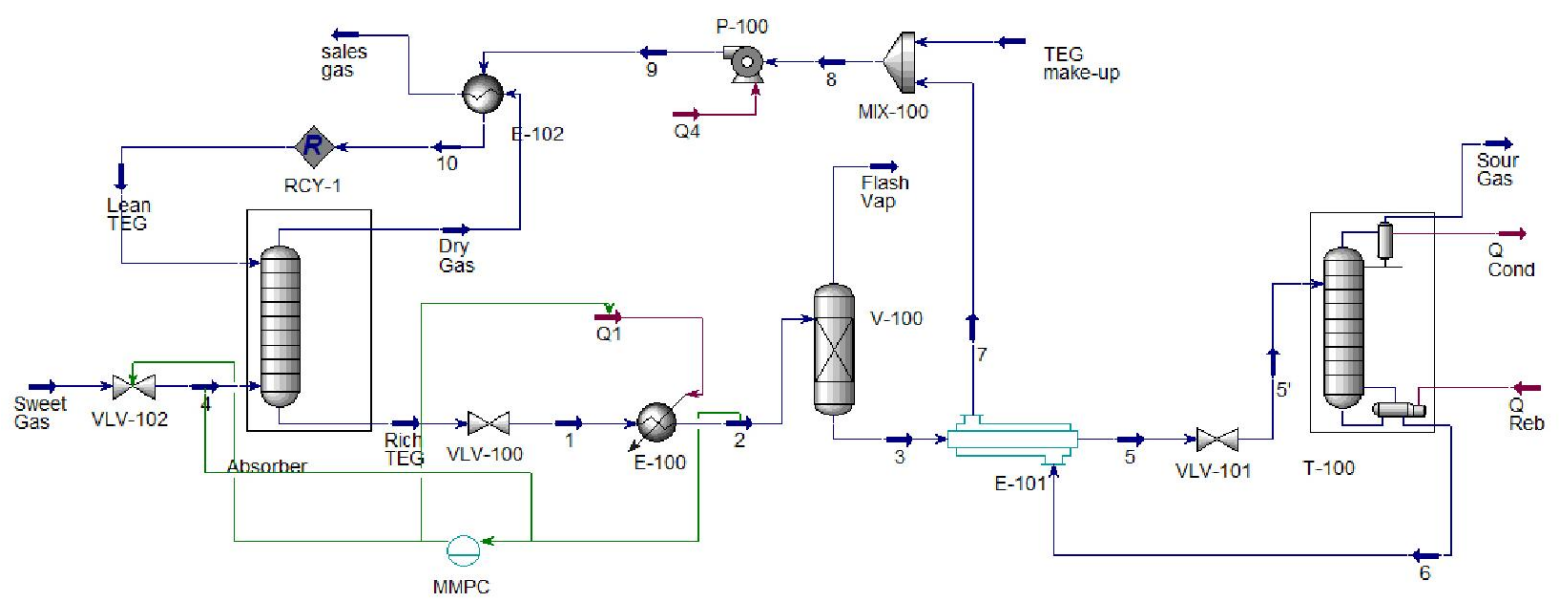

Figure 1. Simulation Design of Gas Dehydration Unit with Multivariable MPC 
For multivariable control systems, the sweet gas flow rate change affects the feed gas pressure and the output temperature of the heater, but changes in the heater heat will affect only the output temperature of the heater, not with the feed gas pressure entering the absorber column. This is because the change in heater heat load has no effect whatsoever on the feed gas pressure entering the absorber column, where the feed gas pressure is affected only by the sweet gas flow rate only. Thus from every change of sweet gas flow rate will be produced 2 PRC, while the heat load heater will be generated 1 PRC, so there are 3 models of FOPDT as in Table 6.

Table 6. FOPDT Model for Multivariable Control

\begin{tabular}{ccc}
\hline Model & CV & MV \\
\hline$G_{1,1}$ & Pressure Stream 4 & Feed gas flow rate (L) \\
\hline$G_{1,2}$ & Pressure Stream 4 & Heater duty (Q1) \\
\hline$G_{2,1}$ & Temperature Stream 2 & Feed gas flow rate (L) \\
\hline$G_{2,2}$ & Temperature Stream 2 & Heater duty (Q1) \\
\hline
\end{tabular}

The $G_{l, 2}$ model cannot be further processed because it has no interaction between the related variables, so only the other three FOPDT models will be processed. All three models of FOPDT are used on Multivariable MPC controllers to control feed gas pressure and heater output temperature multi-input and multi-output. The initial opening of the valve from the sweet gas flow rate is $50 \%$, while the valve initial opening of the heater heat load is $10 \%$, then a step change is made on open loop mode control. The valve opening of the sweet gas flow rate is changed to $60 \%$ (enlarged $10 \%$ ), while the valve opening of the heater heat load is changed to $45 \%$ (35\% magnified). Valve opening changes are based on the effect that such that when the valve aperture is enlarged or reduced by $x$, the value of the $\mathrm{CV}$ due to MV change becomes up or down until the value is stabilized again.

The result of empirical modeling of FOPDT process for pressure and temperature control is shown by Table 7 , with time $(\tau)$ and dead time $(\theta)$ constants in minutes. A larger dead time indicates that the change response due to valve opening changes is slower. Then, the empirical value of process gain $(\mathrm{Kp})$, time constants $(\tau)$ and dead time $(\theta)$ that have been obtained will be input into the settings of the installed Multivariable MPC controller.

Table 7. FOPDT models of TITO system

\begin{tabular}{ccc}
\hline CV & \multicolumn{2}{c}{ MV } \\
\cline { 2 - 3 } & L & Q1 \\
\hline $\begin{array}{c}\text { Feed } \\
\text { pressure }\end{array}$ & $\frac{0.22 e^{-0.06 s}}{0.15 s+1}$ & - \\
\hline $\begin{array}{c}\text { Heater } \\
\text { temperature }\end{array}$ & $\frac{0.925 s^{-077 s}}{0.73 s+1}$ & $\frac{0.47 e^{-0.55 s}}{0.45 s+1}$ \\
\hline
\end{tabular}

\subsection{Multivariable Control MPC Tuning}

Parameters of pressure and temperature controlling with Multivariable MPC are tuned using the Non-Adaptive DMC adjustment strategy developed by Shridhar and Cooper (1998) as well as the fine tuning method in closed loop process. Both methods need to be done to find better Multivariable MPC controller parameters. This is because the method developed by Shridhar and Cooper (1998) is only applicable to distillation column, whereas when the method is used in other operating process unit, the control results are not as good as when applied to the distillation column.

Adjustments with the Non-Adaptive DMC method are performed using the correlations. Meanwhile, the method of fine tuning is done repeatedly until the maximum tuning result is seen based on ISE (Integral Square Error) value which will be calculated later.

Table 8 shows the result of adjusting the Multivariable MPC control parameters for pressure and temperature control. Multivariable MPC fine tuning method is done by trial and error parameters $\mathrm{P}, \mathrm{M}$, and $\mathrm{T}$ to obtain the best control performance based on ISE value.

Table 8. Tuning Parameter of Multivariable MPC

\begin{tabular}{cccc}
\hline Tuning Method & \multicolumn{3}{c}{ Parameter } \\
\cline { 2 - 4 } & $\mathbf{P}$ & $\mathbf{M}$ & $\mathbf{T}$ \\
\hline Non-Adaptive DMC & 58.456 & 18.947 & 2.407 \\
\hline Fine Tuning & 14 & 5 & 3 \\
\hline
\end{tabular}

\subsection{Set Point Tracking}

After obtaining Multivariable MPC controller parameters through both methods above, tested the effect of set point tracking to be able to see the response of control result to controlling parameters of Multivariable MPC that have been done tuning.

The test of the effect of set point change is done on two controlled variables in gas dehydration unit, ie pressure and temperature. For pressure control, the set point is changed by lowering the pressure by $5 \%$ from the initial pressure value and then observing the time required for the process variable to detect any change until it reaches the set point. Figures 2 shows the comparison of pressure control response to a set point change of about 2.4 psi with each adjustment method, both the DMC Non-Adaptive method and the fine tuning method.

Based on Figure 2, it can be seen that the tuning result with fine tuning method is also better when compared with the result of adjustment with NonAdaptive DMC method. It is based on a faster PV value for returning to the set point set after the controller detects any changes to the process by changing the set point. A faster PV value achieving stability indicates that the resulting control parameters are better. It can be seen that using the Non-Adaptive DMC method, the 
time required to achieve stability in the SP value is about 480 seconds, while the PV curve using the fine tuning method takes a shorter time to achieve stability at the SP value, which is about 220 seconds. Of the two PV curves, there is no overshoot indicating that the pressure will not exceed the set point set so it will not pose a hazard due to excessively high operating pressure.

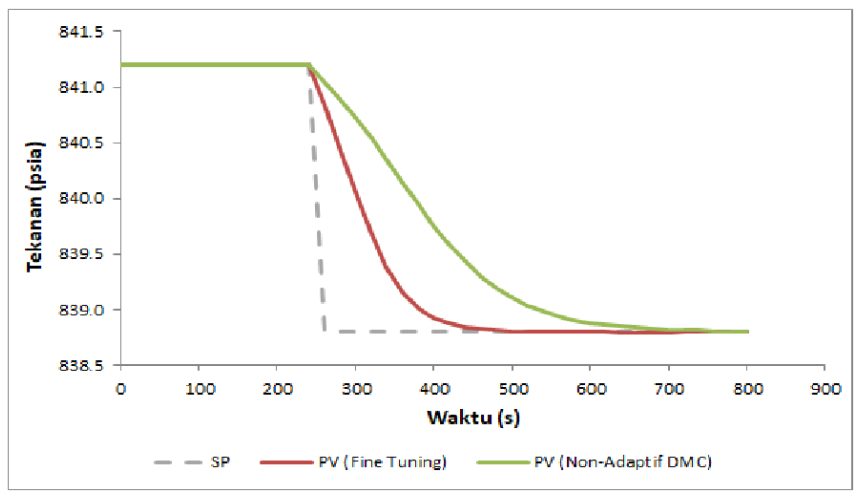

Figure 2. Pressure Response by Comparing Both Tuning Methods

Meanwhile, for temperature control, the set point is changed by raising the temperature by $5 \%$ from the initial temperature value and then also observed the time required for the process variable to detect and then respond to changes to reach the set point. Figures 3 shows the comparison of temperature control response to a 5\% set point change with each adjustment method, both the DMC Non-Adaptive method and the fine tuning method.

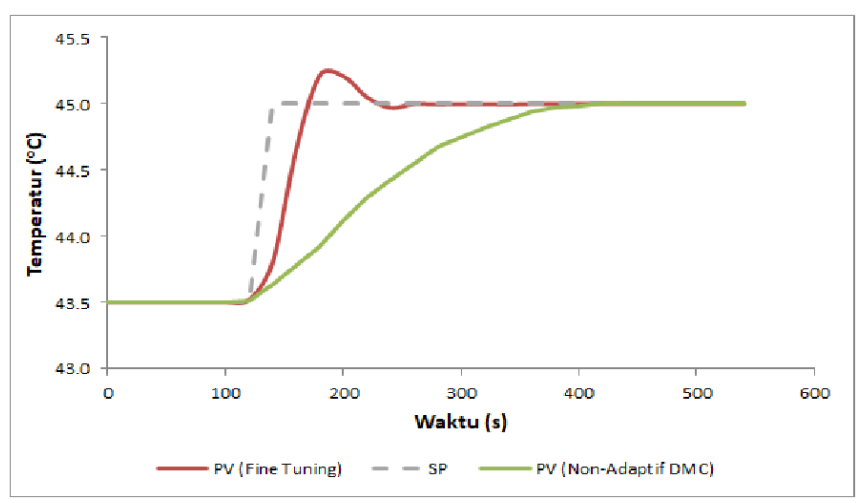

Figure 3. Temperature Response by Comparing Both Tuning Methods

According to Figure 3, it can be seen that the temperature control response which is the result of the tuning by the fine tuning method is also better when compared to the result of the adjustment with the NonAdaptive DMC method, similar to the result obtained in Figure 2 for pressure control. It is based on a faster process temperature variable value to go back to the set point set after the controller responds to a change in the process by changing the set point by $5 \%$ of the initial value. In addition, result of tuning using the fine tuning method gives a faster response when compared to the adjustment constants with the DMC Non-Adaptive method. Although the fine tuning method is dead time and also produces a larger overshoot, it can still be tolerated because the overshoot percentage is still below $25 \%$. In addition, it can be seen also in the fine tuning method has a faster rise time. It also supports the fact that the fine tuning method should be more appropriate to apply.

Another assessment to find out which method is better can be seen based on its ISE value. Table 9 shows the comparison of errors or errors resulting from each controller, both the pressure control and the temperature control.

Table 9. Comparison of Error Values in Pressure and Temperature Control

\begin{tabular}{|c|c|c|}
\hline \multirow{2}{*}{ Tuning Method } & \multicolumn{2}{|c|}{ ISE } \\
\cline { 2 - 3 } & Pressure & Temperature \\
\hline Non-Adaptive DMC & 107.5 & 195 \\
\hline Fine Tuning & 55 & 51 \\
\hline
\end{tabular}

Based on Table 9, it can be seen that the ISE value of the fine tuning method is smaller when compared to the DMC Non-Adaptive method. With smaller ISE values indicating that the control result with the fine tuning method is better than using the Non-Adaptive DMC method.

\subsection{Disturbance Rejection}

The next test is to test the handling of disturbance. Disturbance to be tested is the decrease of feed gas flow rate. The decrease in feed gas flow rate will be set at $5 \%$ of its initial value. From the disturbance, it will be observed the time required for each PV to detect a change until it can return to the set point that has been set. However, in testing this disturbance, only used fine tuning method because it has been proven better to be applied based on the analysis in the previous section. Figures 4 and 5 respectively show the response of pressure and temperature control in handling the disturbance of the feed gas flow rate change.

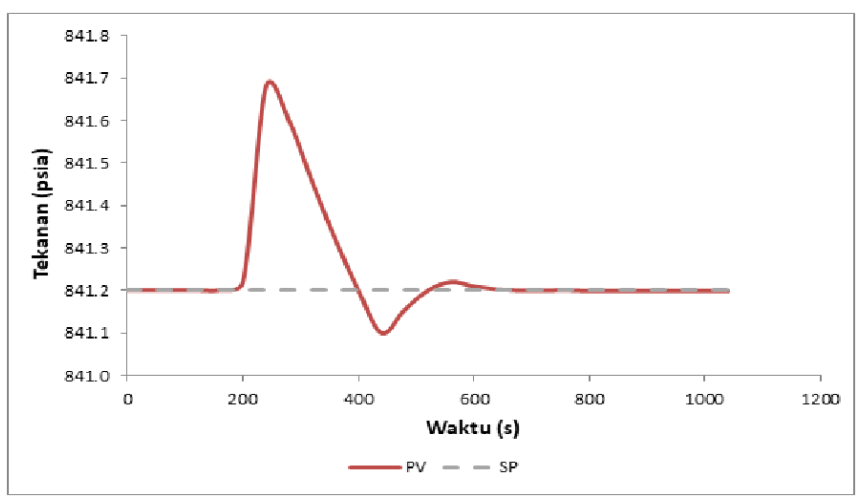

Figure 4. Pressure Response in Handling the Disturbance of the Feed Gas Flow Rate Change

Based on Figure 4 and 5 , it can be seen that Multivariable MPC controller can handle the 
occurrence of disturbance in the form of decreasing feed gas flow rate. Pressure and temperature controls are capable of handling the appearance of disturbance and restore operating conditions at predetermined SP values for approximately 450 seconds. The phenomenon that occurs is the presence of overshoot, with a value that is still at the limit (below 25\%). In addition, oscillations occur on the PV curve but the intensity is not large and is still within $\pm 5 \%$ limit of the specified SP value.

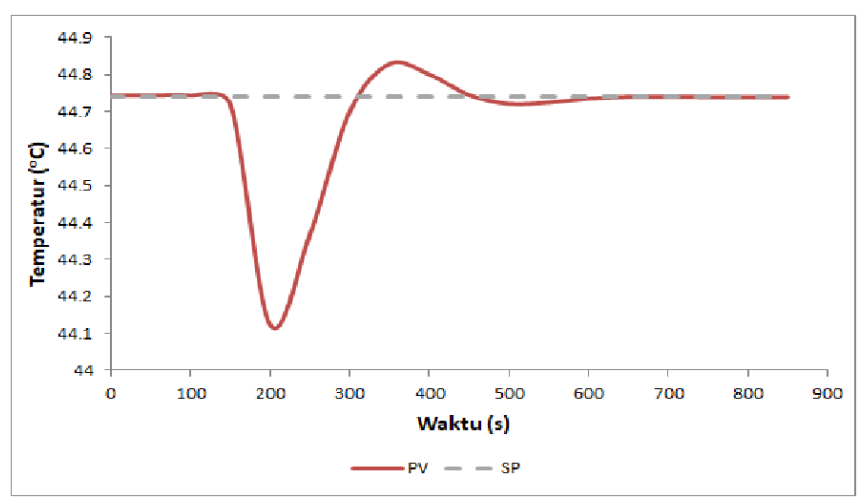

Figure 5. Temperature Response in Handling the Disturbance of the Feed Gas Flow Rate Change

\subsection{Comparison of Multivariable MPC with Proportional Integral}

This section analyzes the control responses between Multivariable MPC and Proportional Integral controllers. Evaluation is done by looking at the response from a faster controller to the set point that has been set. In addition, evaluation is also done based on the value of ISE. Figures 6 and 7 respectively show the comparison of pressure control responses between Multivariable MPC and Proportional Integral controllers.

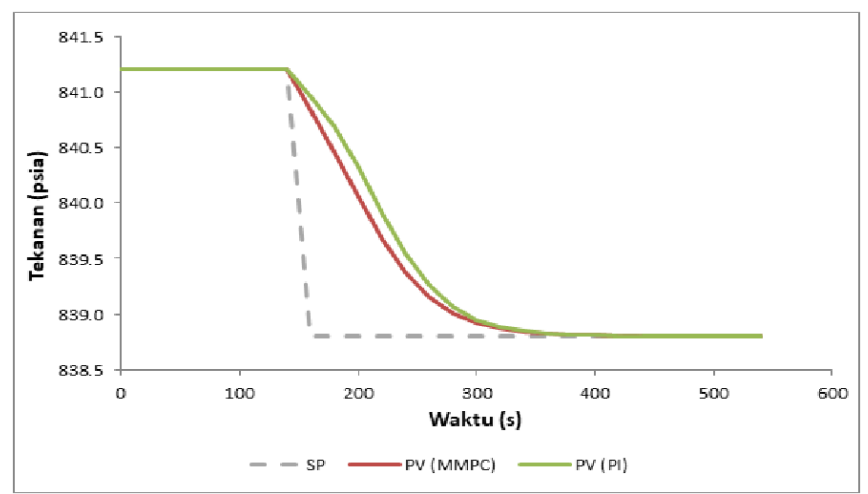

Figure 6. Comparison of Pressure Control Responses between Multivariable MPC With Integral Proportional

Based on Figure 6 and 7, it can be seen that the PV value of the multivariable MPC controller is faster toward the SP value or can be said to achieve stability faster than the PI controller. This makes the Multivariable MPC controller can be said to have a better performance in the gas dehydration process when compared to the PI controller. In pressure control, Multivariable MPC controllers are capable of achieving $\mathrm{SP}$ values in 220 seconds, whereas in PI controllers only reach SP value within 260 seconds. Meanwhile, in temperature control, Multivariable MPC controllers are able to achieve SP value within 140 seconds, while in PI controller only able to reach SP value within 220 seconds.

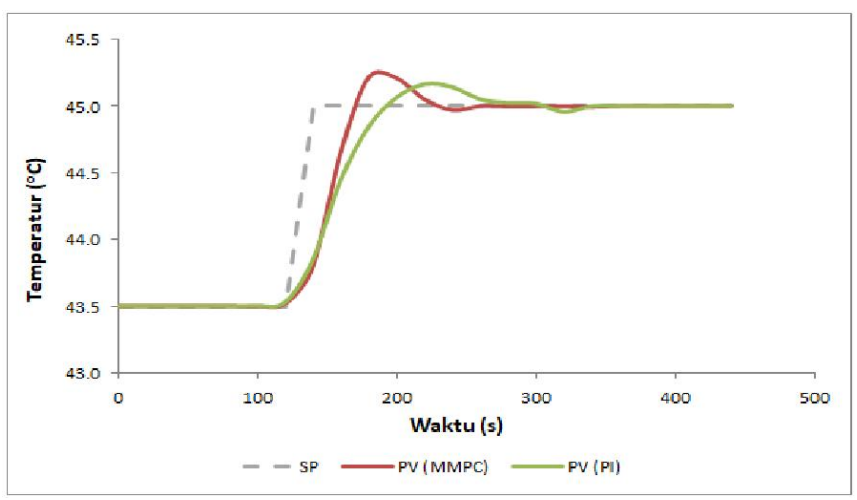

Figure 7. Comparison of Temperature Responses between Multivariable MPC With Integral Proportional

Other assessment to know which type of controller is better can be seen based on its ISE value. Table 10 shows the comparison of error values resulting from each control, both pressure control and temperature control.

Table 10. Comparison of Error Values

\begin{tabular}{ccc}
\hline Controller & \multicolumn{2}{c}{ ISE } \\
\cline { 2 - 3 } Type & Pressure & Temperature \\
\hline PI & 62 & 61 \\
\hline MMPC & 55 & 51 \\
\hline
\end{tabular}

Based on Table 10, it can be seen that the ISE value of the Multivariable MPC is smaller when compared to the ISE value of the PI controller for both controls. With smaller ISE values indicating that the performance of Multivariable MPC controllers can be said to be better than the performance of PI controllers. In addition, by using Multivariable MPC controllers, $11.29 \%$ and $16.39 \%$ control performance improvements were obtained for control and temperature control when compared to PI controllers.

\section{Conclusions}

Based on the results from this study, the following conclusions have been drawn:

- Control system applied to the gas dehydration unit TITO (two-inputs two-outputs) system, ie., sweet gas flow rate and heat duty of heater as $\mathrm{MVs}$, and feed pressure and heater temperature as CVs, respectively. 
- Fine Tuning method is better than method developed by Shridhar and Cooper for the MMPC control of the gas dehydration unit, characterized by smaller ISE values.

- In the SP tracking test, MMPC showed better control performance than the PI controller with $11.29 \%$ performance improvement (pressure control) and $16.39 \%$ (temperature control).

We express our gratitude to the Universitas Indonesia which has funded this research through the scheme of Hibah Publikasi Internasional Terindeks untuk Tugas Akhir Mahasiswa (PITTA) No.2358/UN2.R3.1/HKP.05.00/2018.

\section{References}

1. N. Jacob, Optimization of Tryethylene Glycol (TEG) Dehydration In a Natural Gas Processing Plant. International Journal of Research in Engineering and Technology 3(6): 346-350. (2014).

2. Faramawy, S., Zaki, T., \& Sakr, A.-E. Natural gas origin, composition, and processing: A review. Journal of Natural Gas Science \& Engineering 34: 34-54. (2016).

3. R. Kolass and C. Parker. Moisture Measurement in Natural Gas. Michell Instruments, Ltd., n.d. (2011).

4. P. Kazemi, \& R. Hamidi. Sensitivity Analysis of A Natural Gas Tryethylene Glycol Dehydration Plant In Persian Gulf. Region 53(1) 71-77. ed. (2011).

5. Abdulrahman, R., \& Sebastine, I. Natural Gas Dehydration Process Simulation and Optimization: A Case Study of Khurmala Field in Iraqi Kurdistan Region. (2013).

6. Astr"om, K.J., H“agglund, T., Hang, C.C., and Ho, W.K. Automatic tuning and adaptation for PID controllers - a survey. Cont. Eng. Practice, 1, 699714. (1993).

7. Kano $M$ and Ogawa $M$, The state of the art in chemichal process control in Japan: Good practice and questionnaire survey $J$. Process Contr. 20 969-82.( 2010).

8. A. Wahid and A. Ahmad. Min-max controller output configuration to improve multi-model predictive control when dealing with disturbance rejection. International Journal of Technology. 6 (3): 504-515. (2015).

9. A. Wahid and A. Ahmad . Improved multimodel predictive control to reject very large disturbances on a distillation column. International Journal of Technology. 6: 962971. (2016).

10. Ahmad A. and Wahid A Application of model predictive control (MPC) tuning strategy in multivariable control of distillation column. Reaktor 11(2) 66-70.( 2007).

11. A Wahid and I G E P Putra. Multivariable Model Predictive Control Design of Reactive Distillation Column for Dimethyl Ether
Production. IOP Conf. Series: Materials Science and Engineering 334012018 (2018)

12. Marlin, T. Process Control: Designing Processes and Control Systems for Dynamic Performance. United States: McGraw-Hill Higher Education. (2000).

13. Shridhar, R., \& Cooper, D. J. A Tuning Startegy for Unconstrained Multivariable Model Predictive Control. Control Engineering Practice, 37, 4003 - 4016.(1998). 